\title{
Improving Documentation and Communication Using Operative Note Proformas
}

\author{
Piyush Mahapatra, edmund ieong \\ West Middlesex University Hospital, London
}

\begin{abstract}
Accurate and detailed documentation of surgical procedures is part of good clinical practice, set out by the General Medical Council (GMC). Knee arthroscopy often involves large data sets which require accurate documentation for future assessment and management. This study assesses the quality of documentation of knee arthroscopy, followed by an evaluation of the implementation of a novel operative proforma.
\end{abstract}

A review of 30 consecutive knee arthroscopy operation notes were analysed for missing information, set against a standardised 30 point criteria. An operation proforma was then introduced, and a further 30 consecutive knee arthroscopy operation notes were analysed. We evaluated allied health professional satisfaction with a Likert point scale survey of 21 allied healthcare professionals (recovery and ward nurses, and physiotherapists) following introduction of the proforma. The mean number of missing items on a 30 point scale was 8.8 (range 0 to 23). Examination under anaesthesia was missed in $43 \%$ of cases, tourniquet time in $37 \%$ of cases, and wear results in $17 \%$ of cases. Following introduction of the proforma, the mean number of missing items was 1.1 (range 0 to $24 ; p<0.001$ ). This rose to 3.8 after one year ( $p$ $<0.001$ ) before improvement to $0.7(p<0.01)$ with a new and improved proforma. Eighty percent strongly agreed the operation note was clearer, $90 \%$ strongly agreed it was more legible, $90 \%$ strongly agreed it was more understandable, $50 \%$ strongly agreed there was more information recorded, and $100 \%$ strongly agreed on the proforma having been improved.

Knee arthroscopy is a common procedure with large data sets, which can often be missed or incomplete. A standardised proforma results in a statistically significant improvement in documentation and reduces the incidence of missing information. They are subjectively clearer, more legible, and generally better compared with handwritten notes. This study demonstrates the improvements in healthcare documentation, both clinically and legally, following introduction of a simple proforma. This concept should be applicable to different specialities and procedures in healthcare.

\section{Problem}

The documentation of knee arthroscopy results and intraoperative findings was generally poor in our institution, a busy urban district general hospital. The problem is frequently apparent in outpatient follow up clinics, when it can be difficult to ascertain all of the intraoperative findings, and this can make ongoing management decisions difficult.

Knee arthroscopies are one of the most frequently conducted procedures in orthopaedic practice. The very nature of knee arthroscopies lend themselves to high volume operating lists with rapid patient turnover. Each arthroscopy itself yields a significant amount of information that needs to be recorded. The combination of large amounts of information, coupled with high case volume and rapid turnover, leads to the creation of large data sets, and can often lead to significant omissions and poor quality documentation.

A review of 30 consecutive knee arthroscopy operation notes were analysed for missing information, set against a standardised 30 point criteria. The mean missing items on a 30 point scale was 8.8 (range 0 to 23). Examination under anaesthesia was missed in $43 \%$ of cases, tourniquet time in $37 \%$ of cases, and wear results in $17 \%$ of cases.
As knee arthroscopies are often performed on pooled operating lists, the quality of documentation is vital for ensuring appropriate handover of information from the operating surgeon to the team postoperatively reviewing the patient in the clinic. This can have a significant bearing on patient care, as missing information can lead to inappropriate clinical decisions on ongoing management. For example, failure to appropriately record wear in all compartments may mean that the patient is considered for inappropriate interventions such as unicondylar joint arthroplasty, as opposed to a total knee arthroplasty.

Secondarily, poor quality documentation is a significant barrier to allied health professionals being able to provide the appropriate postoperative treatment, especially if key postoperative instructions are not included in the operation note.

\section{Background}

The poor quality of operation notes is a consistent problem in many institutions, and particularly when considering knee arthroscopies. This has reached an extent where both the Royal College of Surgeons and the GMC have released statements highlighting the importance of accurate and detailed operation notes, in their Good Surgical Practice and Good Medical Practice guides, respectively. 
BMJ Quality Improvement Reports

There have been several varied attempts to try and solve this problem. Perhaps the most widely known is the internet based knee arthroscopy proforma, hosted on www.orthoconsent.com.[1] These proformas have been endorsed by the British Orthopaedic Association (BOA) and include an editable template; however, this requires ready access to IT systems and printing facilities.

Additionally, the website is inaccessible in certain trusts due to stringent IT policies. Documenting operation notes has often proven time consuming to complete, and as such there have been some issues with widespread adoption.

Previously published data has suggested that the use of a preprinted template can significantly improve the quality of documentation.[2]

\section{Baseline measurement}

A retrospective analysis of 30 consecutive knee arthroscopy operation notes were analysed for missing information sets against a standardised 30 point criteria. All patients undergoing simple knee arthroscopy procedures were included, eg diagnostic arthroscopy or partial menisectomy. All patients undergoing complex arthroscopic procedures, eg anterior cruciate ligament reconstruction or meniscal repairs, were excluded. The criteria were selected in conjunction with the senior author, and based on guidelines issued by the BOA. The 30 point criteria is attached for reference (appendix 1).

The mean number of missing items on a 30 point scale was 8.8 (range 0 to 23). Examination under anaesthesia was missed in $43 \%$ of cases, tourniquet time in $37 \%$ of cases, and wear results in $17 \%$ of cases.

See supplementary file: ds5846.pptx - "Appendix 1. 30 point scale of key points of information for knee arthroscopy documentation"

\section{Design}

An operative note proforma was created that included the key criteria as well as a diagrammatic representation that could be annotated by the operating surgeon. The operative proforma was designed using information from www.orthoconsent.com, a freely available online resource endorsed by the BOA.

In our institution, access to IT facilities in the theatre environment can be problematic and inefficient. With the demands of a high turnover operating list, it was thought most effective to have a preprinted proforma that could be stored in the theatre for completion by the operating surgeon immediately post operation.

The proforma was created with the help of one of the senior knee surgeons within the department. We feel that it will be a viable and sustainable solution for the long term, as it is a low cost, low technology intervention that can be implemented easily and will help increase theatre efficiency, as well as having the desired benefit of improving the quality of documentation.

\section{Strategy}

PDSA cycle 1

The proforma was created, and then piloted with two orthopaedic registrars who had had no prior involvement in the project. The viability of the proforma as an operative note was tested. They were asked to document five of their next arthroscopies on the new proforma, in addition to their traditional method of documentation, to test for any errors or provide any suggestions for improvement. The feedback from the testing was generally positive. However, they noted that the use of a non-lateralised image was perhaps slightly misleading. The suggestion to provide lateralised (separate left and right sided) proformas was made. This was incorporated into the new proforma design.

PDSA cycle 2 was then performed, with the same registrars asked to test the new diagram provided on the proforma. The feedback was positive, and the use of lateralised proformas was implemented.

PDSA cycle 3 involved trialling the proforma in one theatre that was commonly used for knee arthroscopies. Post measurement was conducted after completion of PDSA cycle 3.

PDSA cycle 4 involved repeating the audit at one year post implementation of the original proforma. The mean number of missing items had risen to 3.8 , and postoperative weight bearing status was the most frequently missed item. The proforma was modified to include key memory aids. Post measurement was again conducted after PDSA cycle 4

\section{Results}

PDSA cycles 1 and 2 were classified as testing, as the proforma was being developed and refined before being implemented.

After PDSA cycle 3 (single theatre use), a retrospective analysis of 30 consecutive knee arthroscopy operation notes was carried out. The same 30 point scale was used to analyse each operation note for documentation comprehensiveness. The number of items that were missing on each operative note was recorded.

After PDSA cycle 3, the mean number of missing items was 1.1 (range 0 to 24). This was a statistically significant reduction in the mean number of missing items ( $p$ value $<0.001$, Mann-Whitney $U$ test). At one year post cycle 3 (widespread implementation), the mean number of missing items had risen to $3.8(p<0.001)$, with postoperative weight bearing status the most frequently missed item.

Following modification of the proforma in PDSA cycle 4, there was significant improvement in the mean number of missing items, from 3.8 to 0.7 .

In addition, a staff satisfaction survey was conducted after PDSA cycle 4. Fifteen allied health care professionals ( 10 recovery nurses 
and five physiotherapists) directly involved in the immediate aftercare of patients undergoing knee arthroscopy were selected. Each member of staff was asked about their level of experience and banding. They were then asked to give their opinions, using a five point Likert scale, as to whether they felt the new operative proformas were:

1. Clearer

2. More legible

3. Easier to understand

4. Contained more information

5. An improvement to freehand notes.

Ten responses were received (six from recovery staff, and four from physiotherapists). All staff responses received were from full time staff with a wide variety of experience (mean 11.4 years, median 10 years, range 0.5 to 33 years).

All staff members reported that the new proformas were clearer, more legible, more understandable, recorded more information, and were an improvement from freehand written operation notes. Detailed results of the survey are shown in table 2 .

See supplementary file: ds6682.docx - "Table 1 and 2. Mean missing items and healthcare survey results"

\section{Lessons and limitations}

We learned a number of lessons from this project. During the implementation phase it was very important to run a small scale initial trial, to ensure any errors or oversights could be corrected before widespread implementation of a novel system. In our scenario we identified the requirement for lateralised proformas during the initial trial phase.

On a longer term basis, we hope that the implementation of our proforma will provide a clear, robust, and reproducible documentation pathway within our institution. The low cost and easy access system should result in a long, sustainable life for the project. We hope that this will in turn lead to the implementation of further operation notes for procedures that are very standardised, eg total knee arthroplasty.

The intervention is incredibly cost effective, as the printing costs of the novel proformas are negligible. The improvement in documentation will also result in fewer telephone calls between healthcare professionals to clarify missing information, eg weight bearing status or illegible items. This will allow a more efficient and therefore more cost effective arthroscopy service.

Limitations of our study included the relatively small sample size of 30 operation notes. However, the implementation was adequately powered to yield a statistically significant result in documentation improvement.

The sample timeframe was relatively short, and we aim to review the use of the operative proformas in another year to see if the benefits will continue to be sustained, and whether the improvement seen in mean number of missing items between versions one and two of the proforma were due to additional awareness of the operators as a result of the intervention, or if they were a result of the improvement in the proforma.

The staff survey response rate was $66 \%$, and although this does represent a majority, a higher response rate for the survey would have given more definite conclusions.

\section{Conclusion}

Knee arthroscopy is a common procedure with large data sets that can often be missed or incomplete. A standardised proforma results in a statistically significant improvement in documentation and reduces the incidence of missing information. They are subjectively clearer, more legible, and generally better compared with handwritten notes. This study demonstrates the marked improvements in healthcare documentation, both clinically and legally, following introduction of a simple proforma. The results of the staff survey also show that improved documentation significantly improves communication between healthcare professionals, and is very beneficial for integrated multidisciplinary patient care. Staff "buy in" further raises the sustainability of the proforma, as it is now seen as an expectation for the proforma to be present.

This concept should be applicable to different specialties and procedures in healthcare, and we aim to use this project as a platform for the introduction of further proformas for use in standardised procedures, eg total knee arthroplasty.

\section{References}

1. Orthoconsent. www.orthoconsent.com

2. Al-Dadah K, Evans O, Ali F. Optimizing knee arthroscopy documentation using a new template. Br J Hosp Med (Lond) 2014;75(4):227-30

\section{Declaration of interests}

Nothing to declare.

\section{Acknowledgements}

Edmund leong, Robert Ley, Chris Huber.

\section{Ethical approval}

The project was registered with the local audit department and was deemed an improvement project. The project was based on quality of documentation and not a project on human subjects, and therefore formal ethical approval was not required. 